\title{
SAT(ID): Satisfiability of Propositional Logic Extended with Inductive Definitions
}

\author{
Maarten Mariën, Johan Wittocx, Marc Denecker, and Maurice Bruynooghe \\ Department of Computer Science, Katholieke Universiteit Leuven, Belgium \\ \{maartenm, johan, marcd, maurice\}@cs.kuleuven. be
}

\begin{abstract}
We investigate the satisfiability problem, SAT(ID), of an extension of propositional logic with inductive definitions. We demonstrate how to extend existing SAT solvers to become SAT(ID) solvers, and provide an implementation on top of MiniSat. We also report on a performance study, in which our implementation exhibits the expected benefits: full use of the underlying SAT solver's potential.
\end{abstract}

\section{Introduction}

The SAT problem, deciding the satisfiability of propositional logic (PC) theories, has grown very important in recent years. An important research direction is to develop SAT solvers for extensions of PC (e.g., SMT [25]). The use of extended languages leads to broader applicability of SAT-like systems, facilitates the modelling of applications, and may substantially reduce the size of encodings. All of these benefits are true of $\mathrm{PC}(\mathrm{ID})$, the extension of propositional logic with inductive definitions (IDs), as we argue below. In this paper, we present a SAT solver for PC(ID). We call the satisfiability problem of this logic the $S A T(I D)$ problem.

Inductive definitions occur very frequently in real-world problems. A familiar example of an inductive definition is that of reachability. Reachability has so many applications that, for example, the abstract software design analyzer Alloy supports it by a special purpose language construct [12].

$\mathrm{PC}(\mathrm{ID})$ is the propositional fragment of the modelling language $\mathrm{FO}$ (ID) [3, 5]. $\mathrm{FO}(\mathrm{ID})$ is an extension of first order logic with inductive definitions and was proposed as the language of a general declarative problem solving framework based on model expansion [23, 19]. Current solvers reduce FO(ID) model expansion problems to SAT(ID) problems by grounding. Thus techniques and systems as those presented in this paper form the basic inference mechanism of this paradigm.

One approach to the SAT(ID) problem is by encoding the IDs into propositional logic formulas. This approach was followed in [26] but has the disadvantage that it may considerably increase theory size. The alternative approach is by supporting IDs in SAT solvers. In previous work, we developed algorithms for integrating inductive definitions in SAT, and implemented the SAT(ID) solver 
MidL [20,21]. However, these algorithms required changes to the unit propagation mechanism itself, so that we couldn't speak of a proper extension of SAT algorithms. In this work, by contrast, we show how SAT(ID) solvers can be built by extending SAT solvers with an additional propagation mechanism suitable for reasoning on inductive definitions. The obvious advantage from this approach is that the SAT(ID) solver benefits from any improvement made to the underlying SAT solver. In particular, it has the same performance on pure propositional problems as the underlying solver. This was not the case for MidL, which did not have all the fine tuning of a state of the art SAT solver. A further advantage is that by separating the two propagation mechanisms (one for propositional theories and one for IDs), we also strongly simplify the description of a SAT(ID) solver.

We have implemented the technique presented in this paper as an extension of the popular solver MiniSat [7] and have evaluated the system. The resulting solver, MiniSat(ID), is more efficient than our earlier implementation, MidL, and the system of [26]. We also compared MiniSat(ID) with the best solvers of a related formalism, ASP [18], which has a similar expressivity. Though somewhat dependent on the type of problems, the results are positive for MiniSat(ID).

In summary, the contributions of this work are:

- We demonstrate how to extend a SAT solver for SAT(ID). This allows to build SAT(ID) solvers that fully benefit from the best SAT solving technology.

- We strongly simplify the formalisation of a SAT(ID) solver.

- We made an implementation on top of MiniSat that performs substantially better than other SAT(ID) solvers and is at least competitive with ASP solvers.

After formally introducing SAT and SAT(ID) in Section 2, we present a high level overview of the requirements in Section 3, paving the way for the "recipe" for extending a SAT solver with ID propagations, detailed in Section 4. We finish by evaluating the MiniSat(ID) solver in Section 5 .

\section{Preliminaries}

\subsection{Propositional Logic and SAT Solving}

A vocabulary $\Sigma$ is a set of atoms (propositions), a literal is an atom $p$ or its negation $\neg p$. A propositional formula is built from literals using the binary connectives $\vee, \wedge, \equiv, \supset$. A propositional logic theory is a set of formulas. In this paper we assume, without loss of generality, that theories are in conjunctive normal form $(\mathrm{CNF})$ : all formulas are disjunctions of literals, called clauses.

A $\Sigma$-assignment $A$ is a function $A: \Sigma \rightarrow\{\boldsymbol{t}, \boldsymbol{f}, \boldsymbol{u}\}$ ( $\boldsymbol{u}$ stands for "unknown"). An assignment is total when all elements are assigned either $\boldsymbol{t}$ of $\boldsymbol{f}$ and partial otherwise $^{1}$. We leave out $\Sigma$ if it is clear from the context. In this paper we

\footnotetext{
${ }^{1}$ It is more convenient to use a three-valued formalization than to use a partial function that leaves elements undefined when assigned $\boldsymbol{u}$ in the three valued one.
} 
assume that $\Sigma$ always contains the atom $\perp$, which is not used in the theory, and is always assigned $\boldsymbol{f}$. An assignment can be extended to literals: $A(\neg p)=\neg A(p)$ with $\neg \boldsymbol{t}=\boldsymbol{f}, \neg \boldsymbol{f}=\boldsymbol{t}, \neg \boldsymbol{u}=\boldsymbol{u}$, and to clauses and conjunctions of literals: $A(\bigvee C)=\bigvee_{c \in C} A(c)$ with $\bigvee V=\boldsymbol{t}$ if the multiset of truth values $V$ contains $\boldsymbol{t}$, otherwise $\bigvee V=\boldsymbol{u}$ if $V$ contains $\boldsymbol{u}, \bigvee V=\boldsymbol{f}$ otherwise; likewise $A(\bigwedge C)=$ $\bigwedge_{c \in C} A(c)=\neg \bigvee_{c \in C} \neg A(c)$.

A theory $T$ is satisfiable if it has a model: a total assignment $A$ that satisfies every clause of $T$, denoted $A=T$. SAT is the problem of deciding whether a given theory is satisfiable.

SAT solving is the practice of answering the SAT problem. Although other solving techniques exist, the current state of the art solvers are based on DPLL [2] augmented with the two watched literal scheme (2WL) and with clause learning $[24,27]$. The propagation method that drives this search is unit propagation, whereby a literal is assigned true if it occurs as the only non-false literals in a clause. In Section 4.2 we introduce a highly abstracted version of the $2 \mathrm{WL}$ scheme; for more (implementation-oriented) details we refer the reader to [24].

\subsection{Inductive Definitions}

This section is based on work on FO(ID) $[3,5]$. In short, SAT(ID) is the satisfiability problem of the propositional fragment of $\mathrm{FO}$ (ID).

A definition $\Delta$ over vocabulary $\Sigma$ is a set of rules of the form $p \leftarrow \varphi$, where $p \in \Sigma, \leftarrow$ is a new binary connective called definitional implication, and $\varphi$ is a $\Sigma$-formula, called the body of the rule. A theory from propositional calculus (PC) extended with IDs consists of a set of formulas and definitions and is called a PC(ID) theory. In this paper we assume, also without loss of generality [29], that definitions are in definitional normal form (DefNF): for any $p \in \Sigma$, the definition contains at most one rule $p \leftarrow \varphi$, and either $\varphi=\bigvee B_{p}$ or $\varphi=\bigwedge B_{p}$, where $B_{p}$ is a set of literals called the body literals. A PC(ID) theory is DefNF if it contains only one definition, which is in DefNF, and its set of formulas is in CNF. In this paper we also assume that PC(ID) theories are in DefNF.

For a definition $\Delta$, the atoms $\operatorname{Def}(\Delta)=\{p \mid(p \leftarrow \varphi) \in \Delta\}$ are called defined atoms and the others the non-defined ones. Given an assignment $A^{\prime}$ of the non-defined atoms, we can simplify $\Delta$ by substituting these atoms by their truth values. The resulting definition contains only defined atoms and their negative literals; such a set of rules has a unique well-founded model [11], denoted $\operatorname{wfm}_{\Delta}\left(A^{\prime}\right)$. This well-founded model is a (possibly partial) assignment of the defined atoms. A definition $\Delta$ is total (with respect to theory $T$ ) if this wellfounded model $\operatorname{wfm}_{\Delta}\left(A^{\prime}\right)$ is a total assignment (for all assignments $A^{\prime}$ that satisfy $T$ ). Definitions occurring in practice - when bug-free - are always total with respect to the rest of the theory in which they occur ${ }^{2}$, and we restrict

\footnotetext{
${ }^{2}$ For many classes of definitions it is trivial to prove that they are total with respect to any (the empty) theory, e.g. for definitions that do not contain negation.
} 
attention to these unless otherwise said. In Section 4.3 we will explain how to deal with non-total definitions.

A total assignment $A$ satisfies a definition $\Delta$, denoted $A=\Delta$, if $\left.A\right|_{\operatorname{Def}(\Delta)}=$ $\operatorname{wfm}_{\Delta}\left(\left.A\right|_{\Sigma \backslash \operatorname{Def}(\Delta)}\right)$. We say that $A$ extends $\left.A\right|_{\Sigma \backslash D e f(\Delta)}$ to a model of $\Delta$. $A$ satisfies a PC(ID) theory if it satisfies every formula and every definition of the theory. The SAT(ID) problem is the satisfiability problem for PC(ID) theories.

The well-founded semantics correctly formalizes the most common forms of inductive definitions in mathematics: both monotone induction, iterated induction, and non-monotone induction over a well-founded order [6].

Example 1. Consider irreflexive undirected graphs with nodes $\{a, b, c\}$. We represent the edge between nodes $x$ and $y$ by $E_{x y}$. Then $R_{x}$ (for $x \in\{b, c\}$ ) in the following definition expresses the reachability of $x$ from $a\left(I_{x y}\right.$ expresses the reachability of $y$ from $a$ via $x): \Delta_{1}=\left\{R_{b} \leftarrow E_{a b} \vee I_{c b}, \quad R_{c} \leftarrow E_{a c} \vee I_{b c}, \quad I_{c b} \leftarrow\right.$ $\left.R_{c} \wedge E_{b c}, \quad I_{b c} \leftarrow R_{b} \wedge E_{b c}\right\}$.

For instance, to determine reachability from $a$ in a graph with only an edge between $b$ and $c$, we start from the assignment $A^{\prime}$ with $A^{\prime}\left(E_{a b}\right)=A^{\prime}\left(E_{a c}\right)=$ $\boldsymbol{f}$, and $A^{\prime}\left(E_{b c}\right)=\boldsymbol{t}$. Simplifying the definition with $A^{\prime}$ yields $\Delta_{1}^{\prime}=\left\{R_{b} \leftarrow\right.$ $\left.I_{c b}, \quad R_{c} \leftarrow I_{b c}, \quad I_{c b} \leftarrow R_{c}, \quad I_{b c} \leftarrow R_{b}\right\}$. In wfm $\Delta_{1}^{\prime}\left(A^{\prime}\right)$, each of $R_{b}, R_{c}, I_{c b}$ and $I_{c}$ is false, hence the assignment $A_{1}=\left\{E_{a b} \mapsto \boldsymbol{f}, E_{a c} \mapsto \boldsymbol{f}, E_{b c} \mapsto \boldsymbol{t}, R_{b} \mapsto\right.$ $\left.\boldsymbol{f}, R_{c} \mapsto \boldsymbol{f}, I_{c b} \mapsto \boldsymbol{f}, I_{b c} \mapsto \boldsymbol{f}\right\}$ is a model of $\Delta_{1}$.

We will not detail the well-founded semantics here, but refer to [11]. An important property, though, is that $A=\Delta$ implies $A=\Delta[\leftarrow / \equiv]$, where $\Delta[\leftarrow / \equiv]$ is the propositional theory obtained from $\Delta$ by substituting the symbol $\leftarrow$ by $\equiv .^{3}$ The converse is not true; hence $\Delta$ is more constraining (has less models) than $\Delta[\leftarrow / \equiv]$. This means that $\Delta$ will, in general, cause extra constraints that result in extra propagations as compared to $\Delta[\leftarrow / \equiv]$. Precisely the propagation of these extra constraints is what we will add to a SAT solver and is the essence of our contribution.

There exist quadratic algorithms for computing a well-founded model in the context of a given assignment of the non-defined atoms, e.g. [10,17]. However, in the more general context of PC(ID) theories, such assignments are not given.

\section{Requirements for a SAT(ID) Algorithm}

In this section we simplify the formalisation presented earlier in [20]. We start be investigating on an example what are the differences between a definition $\Delta$, and its propositional counterpart $\Delta[\leftarrow / \equiv]$.

Example 2. Example 1 continued. The propositional theory $\Delta_{1}[\leftarrow / \equiv]$ has two models extending $A^{\prime}$ : one where all defined atoms are true, and one where they are all false. However, the first model is not a well-founded model. Indeed,

${ }^{3}$ Rewriting such a substituted rule, $p \equiv \bigvee B_{p}$ or $p \equiv \bigwedge B_{p}$, into CNF is trivial, which is one of the advantages of DefNF. 
analysing the reason for the truth of its defined atoms, one observes a circular reasoning as only possible explanation $\left(R_{b}\right.$ is true because $I_{c b}$ is, $\ldots, I_{b c}$ is true because $R_{b}$ is).

We have to formalize the reasoning about the circularities in the models of $\Delta[\leftarrow / \equiv]$. We do so by using so called justifications, graphical structures that can reveal cycles [4].

Definition 1 (Justification). $A$ justification $J$ for a definition $\Delta$ is a directed graph $(N, E)$, where the nodes $N$ are the literals that occur in $\Delta$ and $E$ is a minimal set of edges satisfying:

- for every rule $d \leftarrow \bigvee B_{d} \in \Delta, E$ contains precisely one edge $(d, b), b \in B_{d}$;

- for every rule $c \leftarrow \bigwedge B_{c} \in \Delta, E$ contains all edges $(c, b), b \in B_{c}$.

The subjustification of a literal $l$ is the subgraph of $J$ consisting of all paths starting in $l$.

We denote the unique descendant in a justification $J$ of a disjunctively defined atom $d$ by $J(d)$. Hence a justification is uniquely characterized by specifying $J(d)$ for each disjunctively defined atom $d$. Note that the edges are directed from defined atoms to body literals.

Example 3. Consider Example 1. For the disjunctive rule defining $R_{b}$ one can choose $J\left(R_{b}\right)$ as either $E_{a b}$ or $I_{c b}$; similarly $J\left(R_{c}\right)$ is either $E_{a c}$ or $I_{b c}$. This results in $2 * 2=4$ possible justifications for $\Delta_{1}$, one of which is shown on the right.

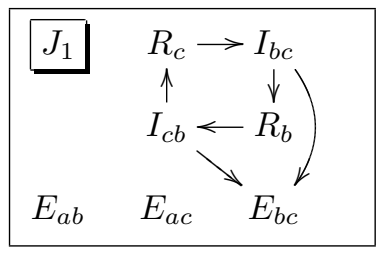

A justification reflects a possible reason for the truth of a defined atom: for a disjunctively defined atom, it suffices that one disjunct is true; for a conjunctively defined atom, all conjuncts are needed.

To formalize the notion that a defined atom is assigned a truth value in accordance with a justification, we introduce the notion of support.

Definition 2 (Support and witness). A justification $J$ supports a (partial) assignment $A$ iff, for each defined atom $p, A(p)=A(\bigwedge\{b \mid(p, b) \in J\})$ when $A(\bigwedge\{b \mid(p, b) \in J\})$ differs from $\boldsymbol{u}$. A supporting justification that contains no cycles (is cycle-free) is a witness for $A$. The subjustification of a defined atom $d$ is a witness of $d$ if it is cycle-free and part of a supporting justification.

Observe that for a total assignment the condition simplifies into: for each defined atom $p, A(p)=A(\bigwedge\{b \mid(p, b) \in J\})$.

Some definitions contain atoms whose truth can never be justified in a wellfounded way (their falsity can); they have to be false in all models. Define $\circlearrowright=$ $\{p \mid$ any subjustification for $p$ contains a cycle $\}$. These atoms have to be false in any model. Defining $\Delta^{\varnothing}$ as the definition obtained by replacing in $\Delta$ the rule $p \leftarrow \varphi$ by $p \leftarrow \perp$ for each $p \in \circlearrowright$ enforces this. It has the advantage of reducing the size of the definition and of simplifying the solver. 
Example 4. Let $\Delta_{4}=\{p \leftarrow q \vee r, \quad q \leftarrow p, \quad r \leftarrow p, \quad s \leftarrow t \vee a, \quad t \leftarrow s\}$. Then $p, q, r$ are defined in terms of each other, so they are part of a cycle in any justification; hence: $\Delta_{4}^{\varnothing}=\{p \leftarrow \perp, \quad q \leftarrow \perp, \quad r \leftarrow \perp, \quad s \leftarrow t \vee a, \quad t \leftarrow s\}$.

Lemma 1. $\Delta \equiv \Delta^{\varnothing}$.

A witness for a (partial) assignment $A$ reflects a well-founded reasoning for the truths in $A$ and the following theorem holds:

Theorem 1. Let $\Delta$ be a total definition, $A$ a total assignment. $A=\Delta$ iff $A=$ $\Delta[\leftarrow / \equiv]$ and there is a justification for $\Delta^{\varnothing}$ that is a witness for $A$.

Example 5. Continuing from Examples 1, 2, 3, we have $\Delta_{1}^{\varnothing}=\Delta_{1}$. We search a model $A_{4}$ of $\Delta_{1}$ that extends $A^{\prime}$. The justification $J_{1}$ with $J_{1}\left(R_{b}\right)=I_{c b}$, $J_{1}\left(R_{c}\right)=I_{b c}$ contains a cycle, hence it cannot be a witness for $A_{4}$. The other justifications have either $J\left(R_{b}\right)=E_{a b}$, or $J\left(R_{c}\right)=E_{a c}$, or both. For any of them to be a witness of $A_{4}$, they should support $A_{4}$, hence we have respectively either $A_{4}\left(R_{b}\right)=\boldsymbol{f}$, or $A_{4}\left(R_{c}\right)=\boldsymbol{f}$, or both. Then because also $A_{4} \models \Delta_{1}[\leftarrow / \equiv]$ is required, $A_{4}\left(R_{b}\right)=A_{4}\left(R_{c}\right)=A_{4}\left(I_{c b}\right)=A_{4}\left(I_{b c}\right)=\boldsymbol{f}$, i.e. $A_{4}=A_{1}$.

In other words, if we have the partial assignment $A^{\prime}$, we can strengthen it (propagate) by making $R_{b}$ and $R_{c}$ false, based on the above reasoning.

The above theorem suggests the following structure for a SAT(ID) algorithm:

- initialize, to find $\Delta^{\varnothing}$,

- apply SAT on $\Delta^{\varnothing}[\leftarrow / \equiv]$ (and on the propositional part of the theory),

- maintain a witness for the assignment found by the SAT solver.

In Section 4 we discuss the details of this strategy.

\section{Recipe for a SAT(ID) Solver}

\subsection{Construction of $\Delta^{\varnothing}$ and of a Cycle-Free Justification}

The purpose of the initialisation step is to identify the atoms of $\circlearrowright$ and to construct $\Delta^{\varnothing}$. We do so by marking literals for which a witness exists. Applying the folowing rules until a fixpoint is reached marks literals with a stratification level.

- Mark all non-defined atoms and all negative literals with 0 .

- Mark an unmarked disjunctively defined atom $d$ with level $l+1$ when one of its body atoms is marked with level $l$

- Mark an unmarked conjunctively defined atom $d$ with level $l+1$ when all of its body atoms are marked with levels $\leq l$.

Atoms that remain unmarked cannot have a witness and belong to $\circlearrowright$. A cyclefree justification of $\Delta^{\varnothing}$ can now easily be derived. For each disjunctively defined atom $d$ of $\Delta^{\varnothing}$ assign to $J(d)$ a literal in the body with a smaller stratification level.

We can mark all negative literals with level 0 because we assume all definitions are total. We come back to that issue in Section 4.3. 


\subsection{Main Search Procedure}

Recall from Section 3 that the goal is to apply SAT on $\Delta^{\varnothing}[\leftarrow / \equiv]$, and to maintain during the search a witness for the current partial assignment. We start with a high level description of the procedure and then elaborate on the details.

The main procedure iterates over the following steps until either a solution is found (a total assignment and a witness for it) or the search space is exhausted.

1. Select a cycle-free justification $J_{c f}$.

2. Use the SAT solver to update the current assignment by performing unit propagation on $\Delta^{\Phi}[\leftarrow / \equiv]$ (and on the propositional part of the theory). (If no propagation is possible, first an undefined atom is assigned $\boldsymbol{t}$ or $\boldsymbol{f}$.)

3. Use the state of the SAT solver to construct a supporting justification $J_{s}$.

4. If $J_{s}$ is not a witness then:

4a Use $J_{c f}$ to adjust $J_{s}$ so that it becomes a witness. In case this fails, a set Cycle is obtained of defined atoms that cannot have a witness under the current assignment (in every supporting justification it holds that the subjustification of the atom has a cycle).

4b If $J_{s}$ is still not a witness, then use $C y c l e$ to extend $\Delta^{\varnothing}[\leftarrow / \equiv]$ with reason clauses, so that propagation in the next iteration will set all atoms in Cycle to false.

Example 6. Let $\Delta_{6}=\{p \leftarrow q \vee r, \quad q \leftarrow p\}$, and let the current assignment have $r$ false, and $p$ and $q$ undefined. A cycle-free justification for $\Delta_{6}$ has $J_{c f}(p)=r$. A supporting justification for $\Delta_{6}$ has $J_{s}(p)=q$ but has a cycle. Hence, $J_{s}(p)$ need to be adjusted. Setting $J_{s}(p)=r$ is not a solution as $J_{s}$ is no longer supporting, hence the adjustment fails and the Cycle-set $\{p, q\}$ is returned. In the next iteration, unit propagation will set $p$ and $q$ to false; the cycle-free justification $J_{c f}$ becomes a supporting one and a solution is obtained.

Step 1: Selection of a cycle-free justification In the first iteration, the cyclefree justification $J_{c f}$ is constructed with the procedure described in Section 4.1 (note that it is a witness as the initial assignment assigns $\boldsymbol{u}$ to all atoms). In later iterations, the most recent witness is used as cycle-free justification, i.e., the supporting justification $J_{s}$ of the previous iteration becomes the cycle-free justification when it is a witness. Taking the most recent witness keeps the difference with the supporting justification to be constructed in step 3 to a minimum and, as will become clear, this reduces the amount of work in step 4a.

Step 2: SAT solving This is a propagation step by the underlying SAT-solver. Note that this includes clause learning and backtracking when propagation leads to the detection of a conflict.

Step 3: Construct a Supporting Justification We assume here that the SAT solver we are extending implements unit propagation using the 2WL scheme. This scheme keeps clauses satisfiable by maintaining for each clause an invariant on its two watched literals. Let $W_{1}(c)$ and $W_{2}(c)$ be the watched literals of 
clause $c$ and $A$ the current assignment, then the invariant is as follows: either $A\left(W_{1}(c)\right)=\boldsymbol{t}$ or $A\left(W_{1}(c)\right)=\boldsymbol{u} \wedge A\left(W_{2}(c)\right) \neq \boldsymbol{f}^{4}$.

We use the watching literals to construct a supporting justification $J_{s}$. This requires to set $J_{s}(d)$ for every disjunctively defined atom $d$. Let $d \leftarrow \bigvee B_{d}$ be the rule defining $d . \Delta[\leftarrow / \equiv]$ contains the clause $\neg d \vee \bigvee B_{d}$; let $W_{1}$ and $W_{2}$ be the matched literals of this clause. If $W_{1}=\neg d$ then $J_{s}(d)=W_{2}$, otherwise $J_{s}(d)=W_{1}$. Knowing that the current assignment is the result of a propagation step, one can easily verify that $J_{s}$ is a supporting justification.

Step 4a: Find a witness The supporting justification $J_{s}$ can have cycles. Because $J_{c f}$ is cycle-free, it must be the case that each cycle in $J_{s}$ contains at least one disjunctively defined atom $d$ with $J_{s}(d) \neq J_{c f}(d)$. Let us call such atoms cycle sources. Cycle sources belong to the set of atoms on which both justifications disagree: $D S=\left\{d \mid J_{s}(d) \neq J_{c f}(d)\right\}$. The overall strategy is to check for each element $c s$ in $D S$ whether it is a cycle source, and, if so, to perform local adjustments on the supporting justification so that $c s$ is no longer part of a cycle ("justifying $c s$ "). Obviously, the smaller $D S$, the less work this step requires.

Note that these local adjustments may or may not update $J_{s}(c s)$ and may update elements in which $J_{s}$ does not differ from $J_{c f}$. The point is that updates break up the cycles passing through $c s$. The reader may wonder whether these updates may introduce new cycles for which no element is present in $D S$. When we explain the details of the adjustments we will argue (inside the description of Analyse $(c s)$ ) that this is not possible. Hence, if all elements of $D S$ can be successfully processed, $J_{s}$ becomes cycle-free and is a witness of the current assignment.

For what concerns the elements $d$ in $D S$ that are false under the current assignment, one can observe that, due to the propagation, all body literals of the rule defining $d$ must be false as well. Hence for such $d, J_{s}$ remains supporting when setting $J_{s}(d)=J_{c f}(d)$ and these elements can be removed from $D S$.

The further processing then consists of justifying each element $c s$ in $D S$ until either $D S$ is empty and hence $J_{s}$ is a witness of the current assignment or some $c s$ could not be justified, in which case a set Cycle as described in the high level algorithm is returned.

Analyse(cs) (A first version of such an algorithm was in [20])

The atom $c s$ is a disjunctively defined atom that is not false in the current assignment and that possibly belongs to a cycle in the supporting justification $J_{s}$.

In an initialisation step, the procedure marks all atoms as unsafe that are on a path in $J_{s}$ that leads to $c s$. This means that all atoms that belong to an eventual cycle are marked as unsafe; however, also other atoms can be marked as unsafe. If $J_{s}(c s)$ is not marked, then there is no cycle passing through $c s$ and we are done. Otherwise, $c s$ is a cycle source and $J_{s}$ has to be adjusted.

\footnotetext{
${ }^{4}$ For other schemes, one first has to use the current assignment to determine for each clause two literals that satisfy the two watching literals invariant.
} 
By "to justify a disjunctively defined atom $d$ " we mean setting $J_{s}(d)$ such that $d$ is no longer part of a cycle through cs; "to justify a conjunctively defined atom" means showing that all its body literals are justified. The purpose of the algorithm is to justify $c s$.

A disjunctively defined atom $d$ can be justified either by setting $J_{s}(d)$ to a literal that is not marked unsafe, or by setting it to an atom that in turn can be similarly justified. To this end, a working queue $\mathcal{Q}$, initialised with $c s$, is maintained: $\mathcal{Q}$ contains atoms that can still be tried to be justified.

The algorithm also maintains a set $C y c l e$, initialised with $c s$, of atoms that are waiting to be justified. If the algorithm fails, then the elements of Cycle have not been justified.

Atoms are popped from $\mathcal{Q}$ and processesed, until either it is ensured that cs is no longer part of a cycle or $\mathcal{Q}$ is empty, in which case Cycle is returned. Let $d$ be the popped element. If it is no longer marked as unsafe, it has already been justified and the next element can be popped. Otherwise, two cases are distinguished. In their description we use a procedure Justify $(q)$ described afterwards.

$d$ is disjunctively defined Let $B_{d}$ be the body of the defining rule. If $B_{d}$ has a literal $b$ that is neither marked nor false, then set $J_{s}(d)=b(d$ is not false under the current assignment, hence, to preserve support, $b$ has to be non false as well) and perform Justify $(d)$. Note that $d$ is now justified: $b$ is not marked and hence has no path to $c s$. Furthermore, it has no path to $d$ either, because all atoms with a path to $d$ in $J_{s}$ are marked. Therefore adding the edge $d \rightarrow b$ cannot create a new cycle. ${ }^{5}$

If all non false literals in $B_{d}$ are marked (they are atoms as negative literals cannot be marked), the ones that are not yet in Cycle are all pushed on $\mathcal{Q}$ and added to Cycle: justifying any of them suffices to justify $d$ (Justify will take care of doing that).

$d$ is conjunctively defined Let $B_{d}$ be the body of the defining rule. If $B_{d}$ has no marked literal, $d$ is justified (not part of a cycle through $c s$ ), so Justify $(d)$ is performed. Otherwise, a marked atom $q$ is selected from $B_{d}$ (preferably one already in $C y c l e$ ) and, if not yet in Cycle, added to it and pushed on $\mathcal{Q}$. This atom $q$ is called the guard of $d$. Adding only this guard to $\mathcal{Q}$ (or none if already in Cycle), instead of all marked body atoms, has the advantage that no computation time will be lost on other body atoms in case this guard cannot be justified. In case it can, Justify $(q)$ will add $d$ to $\mathcal{Q}$ again for reconsideration.

$\operatorname{Justify}(q)$ The "unsafe" mark is removed from the atom $q$ and the atom is removed from Cycle. Moreover, if it is an element from $D S$ then it can be removed from $D S$ as well, as it can no longer be a cycle source. Finally, if it is cs itself, we are done and can start with processing the next element in $D S$. If it is not $c s$, we have to continue:

\footnotetext{
${ }^{5}$ We do not neccessarily have a witness for $b$ (and $d$ ) as $b$ could be part of another cycle with cycle source in $D S$.
} 
- For every disjunctively defined atom $d \in$ Cycle with $q \in B_{d}$, set $J_{s}(d)=q$ and perform Justify $(d)$. Indeed, if $q$ is no longer part of the cycle through $c s$, then so is $d$ in the changed $J_{s}$.

- For every conjunctively defined atom $d$ that has $q$ as guard, $d$ is (again) pushed on $\mathcal{Q}$.

If $\mathcal{Q}$ becomes empty before $c s$ could be justified, some atoms (at least $c s$ itself) are still in Cycle, and all possible supporting subjustifications for them have been exhaustively searched; none has been found that does not cycle through $c s$. This implies that these atoms cannot have a witness (a cycle-free subjustification of a justification supporting the current assignment), hence their truth can never be justified in a well-founded way and it is correct to add the learning clauses in step $4 \mathrm{~b}$.

The use of a queue as datastructure, and not a stack, means that we search for a subjustification of $c s$ that does not cycle through $c s$ by making possible modifications to the supporting justification breadth first, i.e., starting with edges most close to cs.

Step 4b: Learning clauses from the Cycle-set When the supporting justification cannot be adjusted into a cycle-free supporting justification, the set Cycle of defined atoms that cannot have a witness subjustification is returned. These atoms have to be set to false in the current assignment as they necessarily have to be false in each well-founded model extending the current assignment. To properly integrate this with the SAT solver and its backtracking search, this is achieved by extending the theory with an appropriate learned clause for each of these atoms.

Define Ante $=\left(\bigcup_{d \in C y c l e, d \text { disjunctively defined }} B_{d}\right) \backslash C y c l e$, i.e.: all body atoms of the disjunctively defined atoms in the cycle set except the cycle atoms themselves. It is the falsity of those literals that forces the falsity of the atoms in the cycle set. This can be expressed with a so-called loop formula $\bigvee$ Ante $\vee \neg(\bigvee$ Cycle $)$ (adapted from [15]), which in CNF contains one reason clause for each atom in Cycle.

\subsection{Checking Totality of Definitions}

When the search terminates with a SAT model $A$, the existence of the witness $J_{s}$ guarantees (by Theorem 1 ) that $A$ is also a SAT(ID) model (if the definition was total).

Deciding whether a definition is total is as hard as the SAT(ID) problem itself, i.e., NP-hard, hence there is no cheap test that could be used to inform the user if he has written an non-total definition. However, when a model is obtained as above, one can do some verification. One can calculate $\operatorname{wfm}_{\Delta}\left(A^{\prime}\right)$, where $A^{\prime}$ is the restriction of $A$ to the non-defined atoms, and check whether this well-founded model coincides with the original assignment. If it does not, some atoms will be undefined in the well-founded model; it can be pointed out to the user that the definition is not total (the undefined atoms and assignment 
$A^{\prime}$ can help the user in localising the error). Note that coincidence for $A^{\prime}$ does not imply that the definition is total. The well-founded model could be three valued for a different consistent assignment to the non-defined atoms.

\section{Implementation on Top of MiniSat}

\subsection{Introduction}

We have implemented the above algorithms as an extension to the popular SAT solver MiniSat [7]. The resulting program is called MiniSat(ID), and its code is available on [22]. It contains several efficiency improvements compared to the abstracted version as presented here:

- Instead of constructing a supporting justification $J_{s}$ in step 3 and later updating it, we only record the changes with respect to the cycle-free justification $J_{c f}$. When $J_{s}$ eventually becomes a witness, then these changes are applied on $J_{c f}$ in step 1.

- In the initialization, MiniSat(ID) computes strongly connected components (SCCs) in the graph determined by the edges $\left\{(q, b) \mid b\right.$ is an atom of $\left.B_{q}\right\}$. This information can later be used: e.g. to mark as unsafe only atoms in the same SCC as the cycle source.

- When there have been no backtracks since last iteration, finding $D S$ is done by inspecting, using the propagation trail, only those atoms $d$ whose $J_{s}(d)$ has changed.

MiniSat's implementation of $2 \mathrm{WL}$ is particularly suited for finding $J_{s}$ : a clause is stored as an array of literals, with the watches always being the first two in the array, and the first watch never false.

Apart from obvious changes such as command-line options processing, the only change we did to the code of MiniSat proper, was in the "propagate()" method, where we call code to perform steps 3,4 and 1 of our algorithm before completing the method.

\subsection{Evaluation}

SAT(ID) is a new research domain; no organized benchmarking suites exist for it yet. We know of 3 SAT(ID) solvers: idsat [26], MidL [21], and MiniSat(ID). The first works by translating IDs into propositional logic, the second is our earlier work mentioned in the introduction, and the last is a contribution of this paper.

To improve the evaluation, we include also ASP solvers in our comparisons. These can solve the same problems as SAT(ID) solvers, using similar encodings. We have included clasp [8] (which was the winner of the ASP competition in 2007 [9]), SModels ${ }_{c c}$ [28], and cmodels [13]. cmodels uses MiniSat as an underlying SAT solver: it iteratively calls the solver and adds loop formulas until the found SAT model is also an ASP model, or the search space is exhausted. 
We use 2 sets of problem instances with different characteristics: Hamiltonian Cycle problems and Sokoban problems. The full set of encodings and instances that we used and results obtained is available on [22]. Here we provide aggregated results in Tables 1 and $2 .^{6}$

We observe that MiniSat(ID) always performs very similarly to clasp, mostly slightly better. cmodels, on the other hand, shows very variable results. On the Hamiltonian Cycles, it shows its real drawback: whenever there are many cycles in the problem, timings get out of hand, because many iterations are needed. But on Sokoban instances, where reachability is required to express that the agent can reach the square from where his pushing move starts, most squares are reachable most of the time. Consequently, the first model found by the SAT solver underlying cmodels is usually already an ASP model. On these problems, most of the testing for cycles done by solvers as MiniSat(ID) and clasp is redundant.

It is possible to make PC encodings of both Hamiltonian Cycle and Sokoban problems (i.e., encodings that do not require IDs), as well as to automatically translate the PC(ID) instances to PC using idsat. However, both of these approaches yield $\mathrm{PC}$ instances that are orders of magnitude bigger than their corresponding PC(ID) instances. Indeed, using MiniSat on these PC instances yields only timeouts; we have not included these in the tables.

\section{Conclusions and Related Work}

This work is based on earlier work from [20]. In particular, the proof of Theorem 1 in Section 3 is based on a similar theorem there, and the Analyse algorithm from Section 4.2 is in essence the same algorithm as first published there, though the presentation differs considerably. In that paper, the definition of justification was more complex (it involved also justifications for negative literals), and also the concepts $\Delta^{\varnothing}, J_{s}$, and $J_{c f}$ are new to this paper: these changes have enabled us to simplify substantially from [20].

The main contribution of this work, however, is to show how a SAT(ID) solver can be built by extending an existing SAT solver. To do so, we have made the ID propagation mechanism (steps 1, 3 and 4 of our algorithm) as independent as efficiently possible from the unit propagation mechanism: it only requires inspecting the SAT solver's state. By contrast, our earlier solver MidL [21], which was a native SAT(ID) implementation, performed ID related changes to the solver's state during unit propagations.

We have implemented these ideas by extending MiniSat: our experiments show that the resulting solver, MiniSat(ID), exhibits the expected benefits. These experiments included also ASP solvers: ASP is a related formalism with the same expressivity as PC(ID). However, while PC(ID) is simply an extension of propositional logic with the natural concept of inductive definitions, ASP has a more complex relationship with propositional logic [15].

\footnotetext{
${ }^{6}$ Version numbers of all the solvers used: MidL-2.2.1, idsat-0.9.5, clasp-1.0.5, SModels ${ }_{c c^{-}}$ 1.08 (using option "nolookahead" as advised by the authors), cmodels-3.75, MiniSat2.0b. All experiments were run on a P4 $2.8 \mathrm{GHz}$ with $1 \mathrm{~GB}$ memory.
} 


\begin{tabular}{c|ccccc} 
Size & MiniSat(ID) & clasp & \multicolumn{1}{c}{ MidL } & SModels $_{c c}$ & cmodels \\
\hline $200 \times 1800$ & $\mathbf{1 . 2 3}$ & 2.08 & 6.90 & 16.94 & 22.74 \\
$200 \times 2600$ & $\mathbf{1 . 9 4}$ & 3.22 & 6.41 & 38.86 & 35.09 \\
$250 \times 1800$ & 6.41 & $\mathbf{1 . 4 4}$ & $28.06(1)$ & 18.48 & 94.14 \\
$250 \times 2600$ & $\mathbf{2 . 1 9}$ & 2.40 & 24.36 & 30.30 & 92.13 \\
$300 \times 3600$ & $\mathbf{3 . 2 8}$ & 5.61 & 23.81 & 50.60 & $229.54(4)$ \\
$300 \times 4800$ & $\mathbf{4 . 9 0}$ & 6.51 & 35.96 & 79.52 & $178.98(1)$ \\
$350 \times 3600$ & $\mathbf{3 . 6 2}$ & 5.09 & $38.51(2)$ & n/a $(10)$ & $195.91(9)$ \\
$350 \times 4800$ & $\mathbf{5 . 5 3}$ & 6.66 & $50.30(3)$ & n $/$ a (10) & $167.94(7)$
\end{tabular}

Table 1. Evaluation on Hamiltonian Cycle problems: avg. time in sec, (no. of runs $>300 \mathrm{sec}$ ). Averaged over 10 instances.

\begin{tabular}{crccc} 
MiniSat(ID) & clasp & MidL & \multicolumn{1}{c}{ SModels $_{c c}$} & cmodels \\
\hline 7.60 & 7.57 & n/a $(12)$ & 38.26 & $\mathbf{5 . 1 6}$ \\
10.85 & 13.37 & n/a $(12)$ & 63.08 & $\mathbf{8 . 0 2}$ \\
20.33 & 20.59 & n/a $(12)$ & $100.59(1)$ & $\mathbf{1 4 . 3 5}$ \\
56.02 & 57.59 & $223.53(11)$ & $127.40(3)$ & $\mathbf{3 1 . 7 3}$ \\
16.16 & 24.40 & n/a $(12)$ & $69.66(2)$ & $\mathbf{6 . 5 5}$ \\
16.71 & $\mathbf{1 3 . 7 0}$ & $33.2(11)$ & $82.42(2)$ & 24.43 \\
9.65 & 9.68 & $139.65(6)$ & 64.69 & $\mathbf{5 . 7 5}$ \\
34.19 & 34.20 & $219.9(9)$ & $93.76(4)$ & $\mathbf{2 5 . 6 3}$
\end{tabular}

Table 2. Evaluation on Sokoban problems: avg. time in sec, (no. of runs $>300 \mathrm{sec}$ ). Averaged over 12 instances.

\section{References}

1. Chitta Baral, Gerhard Brewka, and John S. Schlipf, editors. Logic Programming and Nonmonotonic Reasoning, 9th International Conference, LPNMR 2007, Proceedings, volume 4483 of Lecture Notes in Computer Science. Springer, 2007.

2. Martin Davis, George Logemann, and Donald W. Loveland. A machine program for theorem-proving. Commun. ACM, 5(7):394-397, 1962.

3. Marc Denecker. Extending classical logic with inductive definitions. In Lloyd et al. [16], pages 703-717.

4. Marc Denecker and Danny De Schreye. Justification semantics: A unifiying framework for the semantics of logic programs. In Luís Moniz Pereira and Anil Nerode, editors, LPNMR, pages 365-379. MIT Press, 1993.

5. Marc Denecker and Eugenia Ternovska. A logic of non-monotone inductive definitions and its modularity properties. In Lifschitz and Niemelä [14], pages 47-60.

6. Marc Denecker and Joost Vennekens. Well-founded semantics and the algebraic theory of non-monotone inductive definitions. In Baral et al. [1], pages 84-96.

7. Niklas Eén and Niklas Sörensson. An extensible SAT-solver. In Enrico Giunchiglia and Armando Tacchella, editors, SAT, volume 2919 of Lecture Notes in Computer Science, pages 502-518. Springer, 2003.

8. Martin Gebser, Benjamin Kaufmann, André Neumann, and Torsten Schaub. clasp: A conflict-driven answer set solver. In Baral et al. [1], pages 260-265.

9. Martin Gebser, Lengning Liu, Gayathri Namasivayam, André Neumann, Torsten Schaub, and Miroslaw Truszczynski. The first answer set programming system competition. In Baral et al. [1], pages 3-17. 
10. Allen Van Gelder. The alternating fixpoint of logic programs with negation. Journal of Computer and System Sciences, 47(1):185-221, 1993.

11. Allen Van Gelder, Kenneth A. Ross, and John S. Schlipf. The well-founded semantics for general logic programs. J. ACM, 38(3):620-650, 1991.

12. Daniel Jackson. Software Abstractions: Logic, Language, and Analysis. MIT Press, Cambridge, MA, 2006.

13. Yuliya Lierler. cmodels - SAT-based disjunctive answer set solver. In Chitta Baral, Gianluigi Greco, Nicola Leone, and Giorgio Terracina, editors, LPNMR, volume 3662 of Lecture Notes in Computer Science, pages 447-451. Springer, 2005.

14. Vladimir Lifschitz and Ilkka Niemelä, editors. Logic Programming and Nonmonotonic Reasoning, 7th International Conference, LPNMR 2004, Proceedings, volume 2923 of Lecture Notes in Computer Science. Springer, 2004.

15. Fangzhen Lin and Yuting Zhao. ASSAT: Computing answer sets of a logic program by sat solvers. Artif. Intell., 157(1-2):115-137, 2004.

16. John W. Lloyd et al.i, editors. Computational Logic - CL 2000, First International Conference, Proceedings, volume 1861 of LNCS. Springer, 2000.

17. Zbigniew Lonc and Mirosław Truszczyński. On the problem of computing the well-founded semantics. In Lloyd et al. [16], pages 673-687.

18. Victor W. Marek and Mirosław Truszczyński. Stable models and an alternative logic programming paradigm. In Krzysztof R. Apt, Victor W. Marek, Mirosław Truszczyński, and David S. Warren, editors, The Logic Programming Paradigm: a 25-Year Perspective, pages 375-398. Springer-Verlag, 1999.

19. Maarten Mariën, Johan Wittocx, and Marc Denecker. The IDP framework for declarative problem solving. In Search and Logic: Answer Set Programming and SAT, pages 19-34, 2006.

20. Maarten Mariën, Johan Wittocx, and Marc Denecker. Integrating inductive definitions in SAT. In Nachum Derschowitz and Andrei Voronkov, editors, LPAR, volume 4790 of Lecture Notes in Computer Science, pages 378-392. Springer, 2007.

21. Maarten Mariën, Johan Wittocx, and Marc Denecker. MidL: a SAT(ID) solver. In 4th Workshop on Answer Set Programming: Advances in Theory and Implementation, pages 303-308, 2007.

22. http://www.cs.kuleuven.be/ dtai/krr/software/minisatid.html.

23. David G. Mitchell and Eugenia Ternovska. A framework for representing and solving NP search problems. In Manuela M. Veloso and Subbarao Kambhampati, editors, AAAI, pages 430-435. AAAI Press / The MIT Press, 2005.

24. Matthew Moskewicz, Conor Madigan, Ying Zhao, Lintao Zhang, and Sharad Malik. Chaff: Engineering an efficient SAT solver. In $D A C^{\prime} 01$, pages 530-535. ACM, 2001.

25. Robert Nieuwenhuis, Albert Oliveras, and Cesare Tinelli. Solving SAT and SAT modulo theories: From an abstract Davis-Putnam-Logemann-Loveland procedure to DPLL $(t)$. J. ACM, 53(6):937-977, 2006.

26. Nikolay Pelov and Eugenia Ternovska. Reducing inductive definitions to propositional satisfiability. In Maurizio Gabbrielli and Gopal Gupta, editors, ICLP, volume 3668 of Lecture Notes in Computer Science, pages 221-234. Springer, 2005.

27. João P. Marques Silva and Karem A. Sakallah. GRASP: A search algorithm for propositional satisfiability. IEEE Trans. Computers, 48(5):506-521, 1999.

28. Jeffrey Ward and John S. Schlipf. Answer set programming with clause learning. In Lifschitz and Niemelä [14], pages 302-313.

29. Johan Wittocx, Joost Vennekens, Maarten Mariën, Marc Denecker, and Maurice Bruynooghe. Predicate introduction under stable and well-founded semantics. In Sandro Etalle and Mirosław Truszczyński, editors, ICLP, volume 4079 of Lecture Notes in Computer Science. Springer, 2006. 